\title{
Alteration of the oral environment in patients undergoing esophagectomy during the perioperative period
}

\author{
Masami YOSHIOKA ${ }^{1}$, Daisuke HINODE ${ }^{2}$, Yota YAMAMOTO ${ }^{3}$, Yoshihito FURUKITA ${ }^{4}$, Akira TANGOKU ${ }^{5}$
}

\begin{abstract}
1- DDS, PhD, Associate Professor, Department of Oral Health and Welfare, Institute of Health Biosciences, The University of Tokushima Graduate School, Tokushima, Japan.

2- DDS, PhD, Professor, Department of Hygiene and Oral Health Science, Institute of Health Biosciences, The University of Tokushima Graduate School, Tokushima, Japan.

3- MD, PhD, Assistant Professor, Department of Thoracic, Endocrine Surgery and Oncology, Institute of Health Biosciences, the University of Tokushima Graduate School, Tokushima, Japan.

4- MD, Assistant Professor, Department of Thoracic, Endocrine Surgery and Oncology, Institute of Health Biosciences, the University of Tokushima Graduate School, Tokushima, Japan.

5- MD, PhD, Professor, Department of Thoracic, Endocrine Surgery and Oncology, Institute of Health Biosciences, the University of Tokushima Graduate School, Tokushima, Japan.
\end{abstract}

Corresponding address: Masami Yoshioka - Department of Oral Health and Welfare, Institute of Health Biosciences, The University of Tokushima Graduate School, Tokushima - 770-8504, Japan - Phone: +81 886337543 - fax: +81 886337898 - e-mail: masami@dent.tokushima-u.ac.jp

Received: May 9, 2012 - Modification: January 4, 2013 - Accepted: January 18, 2013

\section{ABSTRACT}

\begin{abstract}
bjective: During the perioperative period, oral ingestion is changed considerably in esophagectomy patients. The aim of this study was to investigate oral environment modifications in patients undergoing esophageal cancer treatments due to changes in dietary intake and swallowing functions. Material and Methods: Thirty patients who underwent operation for removal of esophageal cancer in Tokushima University Hospital were enrolled in this study. Results: It was found that 1 ) the flow rate of resting saliva decreased significantly at postoperative period by deprived feeding for one week, although it did not recover several days after oral ingestion began, 2 ) the accumulation of dental plaque and the number of mutans streptococci in saliva decreased significantly after operation, while both increased relatively quick when oral ingestion began, and 3 ) the swallowing function decreased significantly in the postoperative period. Conclusions: These results suggest that dental professionals should emphasize the importance of oral health care and provide instructions on plaque control to patients during the perioperative period of esophageal cancer treatment.
\end{abstract}

Key words: Oral environment. Esophageal cancer. Perioperative period.

\section{INTRODUCTION}

Currently, cancer treatments are increasingly more effective, although they have been associated with short and long term oral side effects such as oral candidiasis ${ }^{6}$ and oral mucositis ${ }^{21}$. Regarding esophageal cancer, it has been reported that the most frequent problems associated with esophageal surgery were related to respiratory complications, which result in increased critical care stay, hospital stay and mortality ${ }^{13}$. In addition, Atkins and $D^{\prime} A m i c{ }^{2}$ (2006) reported that pulmonary complications were the most common source of morbidity and mortality after esophageal resection. In addition, Akutsu, et al. ${ }^{1}$ (2010) reported that frequent preoperative dental brushing prevented postoperative pneumonia in esophageal cancer patients. The above observations suggest that an efficient control of oral microorganisms may be very important to provide an excellent prognosis for the esophageal surgery.

Malnourished patients undergoing major surgical treatments are at a high risk of morbidity and mortality, and therefore identification and treatment for malnutrition is of utmost importance ${ }^{15}$. Seike, et al. ${ }^{17}$ (2011) suggested that perioperative 
nutritional support can be safely performed either with total parenteral nutrition (TPN) or enteral nutrition (EN). EN is now used more frequently for nutritional support after resection for esophageal cancer. Fujita, et al. ${ }^{10}$ (2012) showed that early EN reduces the incidence of life-threatening surgical complications and improves the completion rate of the clinical pathway for thoracic esophagectomy. It has been reported that postoperative EN associated with arginine, RNA, and omega-3 fatty acids supplements significantly improved immunologic, metabolic and clinical outcomes in patients with upper gastrointestinal malignancies who were undergoing a major elective surgery ${ }^{7}$. When applying orally a high-density liquid diet whose nutrients are similar to those of EN to a patient before an operation, the oral environment changes considerably. The patients have to abstain from solid food until about 1 week after operation, meanwhile intaking liquid foods, and then they are released to eat solid foods. The situation of the oral ingestion is considerably modified for the patient after esophagectomy. Therefore, alterations in the oral environment of patients will occur during these perioperative periods.

It seems important for the patient comfort to maintain a healthy environment in the simplest possible manner in order to give patients the best chance for a rapid and uncomplicated recovery. Although oral health care for patients receiving cancer treatments is very important ${ }^{16,22}$, there are few studies regarding the oral environment at esophagectomy. A clinical study conducted on hyposalivation in hospitalized patients suggested that clinical diagnosis of hyposalivation might be helpful to provide an immediate and appropriate therapy avoiding further problems and improving their quality of life ${ }^{5}$. Therefore, it is necessary to determine the influence of oral health conditions in perioperative patients and to construct an effective protocol of oral health care due to the change of nutrition status or dietary intake. In this study, we investigated the impact of a high-density liquid diet in patients having received an esophageal cancer treatment by the determination of salivary flow rate and use of caries activity tests.

\section{MATERIAL AND METHODS}

\section{Study subjects and protocol}

Thirty patients aged between 52 and 81 (23 males and 7 females, mean age: $64.9 \pm 7.2$ years), who underwent esophagectomy for thoracic esophageal cancer at the Tokushima University Hospital from February 2010 to January 2012 were enrolled in the study (Figure 1), which had been previously approved by the Ethics Committee of Tokushima University Hospital (protocol approval:
897). Prior to enrolment, the participants were informed about the methods and objectives of the study and they provided written informed consent.

The study protocol is summarized in Figure 2. Clinical parameters assessments, saliva collection and caries activity tests were generally performed at bed-side between 16:00 and 18:00 ( $\geq 3 \mathrm{~h}$ after lunch) to avoid any influence associated with the last meal. Subjects took orally the high-density liquid diet (ANOM; Otsuka Pharmaceutical Co, Ltd., Tokushima, Japan $)^{12}$, which included L-arginine, DNA and omega-3 fatty acids, in order to boost the immune system from approximately 5 days before the operation, in addition to the regular meal. Nutrition management during the fasting period after the esophageal surgery was supported by TPN or EN via an enteral feeding tube inserted from jejunostomy during the operation. They could start intake of the postoperative liquid diet around 8 days after checking that there was no disclosure and no infection at the operative site.

In this project, as summarized in Figure 2, the oral cavity of the subjects was investigated at 5 examination periods, as follows: the period of usual dietary intake (4-7 days before an operation: E1), the period of usual meal and high density liquid diet ingestion (3-4 days before an operation: E2), the period before the liquid diet ingestion starts (7-9 days after an operation: E3), the period of ingestion of semiliquid foods using rice porridge (10-15 days after an operation: E4), the period of ingestion of soft solids (15-23 days after an operation: E5). We named the fasting period after the esophageal surgery as NPO (Nil per os) and the postoperative day as POD.

\section{Clinical parameters related to the oral environment \\ Plaque Index ${ }^{18}$ (PI) and Gingival Index ${ }^{11}$ (GI)} were recorded as clinical parameters of the oral environment. In order to investigate the status of the swallowing function at each examination period, the repetitive saliva swallowing test (RSST) was performed, which determines how many times a subject can swallow his own saliva in $30 \mathrm{~s}^{4}$.

\section{Determination of saliva flow rate and caries activity tests}

Non-stimulated whole saliva (resting saliva) was obtained by spitting or direct expectoration while stimulated saliva was harvested by chewing a paraffin pellet. Both saliva samples were collected in sterile plastic tubes for $5 \mathrm{~min}$ and for $3 \mathrm{~min}$, respectively, prior to assessing the clinical parameters and the volume was measured. The edentulous subjects and/or those lacking teeth in chewing sites were excluded in the evaluation of stimulated saliva. Dentocult SM Strip mutans test (Orion Diagnostica 
Ltd., Espoo, Finland) that determines the number of mutans streptococci in saliva was carried out according to the manufacturer's instructions.

CAT21 test (Willdent Co Ltd., Osaka, Japan) that shows the acid production ability of dental plaque reflecting the number of acid-producing oral bacteria such as mutans streptococci and/ or lactobacilli was performed according to the manufacturer's instructions. An examiner swabbed dental plaque on buccal surface of all erupted maxillary teeth using a cotton bud and inserted it into a tube containing the diagnostic reagents. After incubation at $37^{\circ} \mathrm{C}$ for $48 \mathrm{~h}$, the color change in the tube was compared with the color chart, which assesses the levels of acid production. Test scores ranged in 4 levels depending on the lactic acid formation rate. Blue color (-) indicated a low formation rate of lactic acid, $\mathrm{pH}$ 7.2-5.7 and green color $(+)$ indicated a medium formation rate, $\mathrm{pH}$ 5.7-5.0. Yellow green color $(++)$ and yellow color $(+++)$ indicated a high production of lactic acid, $\mathrm{pH}$ 5.0-4.4 and $\mathrm{pH} 4.4-3.8$, respectively.

\section{Statistical analysis}

Statistical significance in flow rate of both resting and stimulated saliva was assessed by a paired Student's t-test. Other clinical parameters and caries activity tests were assessed by a Wilcoxon's signed-ranks test using the statistical software Statcel2 integrated into Microsoft Excel for Windows (The publisher OMS Ltd., Tokyo, Japan). Differences were considered statistically significant at $p<0.05$. Since the number of subjects for whom the availability of the data of 5 periods by each method was restricted (Figure 1), statistical comparison between 2 different periods was performed using available data corresponding to 2 periods by each method.

\section{RESULTS}

\section{Alteration of salivary flow rates}

Figure 3 reports the changes in flow rate of resting saliva of 11 subjects (shown as open circle in Figure 1) and stimulated saliva of 7 subjects (shown as closed circle in Figure 1 ) at the 5 examination periods. The mean salivary flow rate \pm S.D. of

\begin{tabular}{|c|c|c|c|c|c|c|c|c|c|c|c|c|c|c|c|c|c|c|c|c|c|c|c|c|c|c|c|c|}
\hline \multirow{2}{*}{$\begin{array}{l}\text { Subject } \\
\text { number }\end{array}$} & \multirow{2}{*}{ Age } & \multirow[b]{2}{*}{ Sex } & \multirow{2}{*}{$\begin{array}{l}\text { Number } \\
\text { of teeth }\end{array}$} & \multicolumn{5}{|c|}{ Resting saliva } & \multicolumn{5}{|c|}{ Stimulated saliva } & \multicolumn{5}{|c|}{ PVGI } & \multicolumn{5}{|c|}{ Dentocult SM } & \multicolumn{5}{|c|}{ CAT21 } \\
\hline & & & & E1 & E2 & E3 & E4 & E5 & E1 & E2 & E3 & E4 & E5 & E1 & E2 & E3 & E4 & E5 & E1 & E2 & E3 & E4 & E5 & E1 & E2 & E3 & E4 & E5 \\
\hline 1 & 54 & $\mathrm{~F}$ & 29 & & $\bullet$ & $\bullet$ & $\bullet$ & & & $\bullet$ & $\bullet$ & $\bullet$ & & & $\bullet$ & $\bullet$ & $\bullet$ & & & $\bullet$ & $\bullet$ & $\bullet$ & & & $\bullet$ & $\bullet$ & $\bullet$ & \\
\hline 2 & 52 & M & 29 & - & & - & - & - & - & & - & - & - & - & & - & - & - & • & & - & - & - & - & & - & - & - \\
\hline 3 & 60 & $\mathrm{~F}$ & 24 & $\bullet$ & & & & & $\bullet$ & & & & & $\bullet$ & & & & & $\bullet$ & & & & & $\bullet$ & & & & \\
\hline 4 & 63 & M & 9 & - & $\bullet$ & & $\bullet$ & $\bullet$ & & & & & & $\bullet$ & $\bullet$ & & - & - & $\bullet$ & $\bullet$ & & $\bullet$ & $\bullet$ & $\bullet$ & - & & $\bullet$ & $\bullet$ \\
\hline 5 & 61 & M & 21 & & $\bullet$ & & $\bullet$ & $\bullet$ & & - & & - & $\bullet$ & & $\bullet$ & & $\bullet$ & $\bullet$ & & $\bullet$ & & $\bullet$ & $\bullet$ & & $\bullet$ & & $\bullet$ & $\bullet$ \\
\hline 6 & 53 & M & 29 & 0 & 0 & 0 & 0 & 0 & $\bullet$ & $\bullet$ & $\bullet$ & & $\bullet$ & $\bullet$ & $\bullet$ & $\bullet$ & & $\bullet$ & $\bullet$ & & $\bullet$ & & & 0 & 0 & 0 & 0 & 0 \\
\hline 7 & 60 & M & 27 & 0 & 0 & 0 & 0 & 0 & 0 & 0 & 0 & 0 & 0 & 0 & 0 & 0 & 0 & 0 & & - & $\bullet$ & - & - & 0 & 0 & 0 & 0 & 0 \\
\hline 8 & 73 & $\mathrm{~F}$ & 28 & $\bullet$ & $\bullet$ & & $\bullet$ & & $\bullet$ & $\bullet$ & & $\bullet$ & & $\bullet$ & $\bullet$ & & $\bullet$ & & $\bullet$ & $\bullet$ & & $\bullet$ & & $\bullet$ & $\bullet$ & & & \\
\hline 9 & 65 & M & 8 & $\bullet$ & & & & & - & & & & & $\bullet$ & & & & & $\bullet$ & & & & & $\bullet$ & & & & \\
\hline 10 & 64 & M & 21 & - & - & $\bullet$ & & & $\bullet$ & - & $\bullet$ & & & $\bullet$ & $\bullet$ & - & & & - & - & & & & $\bullet$ & $\bullet$ & $\bullet$ & & \\
\hline 11 & 60 & M & 28 & $\bullet$ & $\bullet$ & $\bullet$ & - & & $\bullet$ & $\bullet$ & $\bullet$ & - & & $\bullet$ & $\bullet$ & $\bullet$ & - & & $\bullet$ & $\bullet$ & - & • & & $\bullet$ & $\bullet$ & $\bullet$ & - & \\
\hline 12 & 68 & M & 3 & & $\bullet$ & $\bullet$ & $\bullet$ & & & & & & & & $\bullet$ & $\bullet$ & $\bullet$ & & & $\bullet$ & $\bullet$ & $\bullet$ & & & $\bullet$ & $\bullet$ & $\bullet$ & \\
\hline 13 & 61 & M & 26 & 0 & 0 & 0 & 0 & 0 & 0 & 0 & 0 & 0 & 0 & 0 & 0 & 0 & 0 & 0 & 0 & 0 & 0 & 0 & 0 & 0 & 0 & 0 & 0 & 0 \\
\hline 14 & 66 & $\mathrm{M}$ & 29 & $\bullet$ & $\bullet$ & & & & $\bullet$ & $\bullet$ & & & & $\bullet$ & $\bullet$ & & & & $\bullet$ & $\bullet$ & & & & $\bullet$ & $\bullet$ & & & \\
\hline 15 & 60 & $\mathrm{~F}$ & 27 & $\bullet$ & $\bullet$ & $\bullet$ & $\bullet$ & & $\bullet$ & $\bullet$ & $\bullet$ & $\bullet$ & & $\bullet$ & $\bullet$ & $\bullet$ & $\bullet$ & & $\bullet$ & $\bullet$ & $\bullet$ & $\bullet$ & & $\bullet$ & $\bullet$ & $\bullet$ & $\bullet$ & \\
\hline 16 & 68 & $M$ & 28 & 0 & 0 & 0 & 0 & 0 & 0 & 0 & 0 & 0 & 0 & 0 & 0 & 0 & 0 & 0 & 0 & 0 & 0 & 0 & 0 & 0 & 0 & 0 & 0 & 0 \\
\hline 17 & 71 & $\mathrm{M}$ & 22 & $\bullet$ & $\bullet$ & $\bullet$ & & $\bullet$ & & & & & $\bullet$ & 0 & 0 & 0 & 0 & 0 & 0 & 0 & 0 & 0 & 0 & 0 & 0 & 0 & 0 & 0 \\
\hline 18 & 69 & $M$ & 22 & 0 & 0 & 0 & 0 & 0 & 0 & 0 & 0 & 0 & 0 & 0 & 0 & 0 & 0 & 0 & 0 & 0 & 0 & 0 & 0 & 0 & 0 & 0 & 0 & 0 \\
\hline 19 & 56 & $M$ & 27 & $\bullet$ & & $\bullet$ & $\bullet$ & $\bullet$ & $\bullet$ & & $\bullet$ & $\bullet$ & $\bullet$ & $\bullet$ & & $\bullet$ & $\bullet$ & $\bullet$ & $\bullet$ & & $\bullet$ & $\bullet$ & $\bullet$ & $\bullet$ & & $\bullet$ & $\bullet$ & $\bullet$ \\
\hline 20 & 79 & $\mathrm{~F}$ & 28 & $\bullet$ & $\bullet$ & & & & $\bullet$ & $\bullet$ & & & & $\bullet$ & $\bullet$ & & & & $\bullet$ & $\bullet$ & & & & $\bullet$ & $\bullet$ & & & \\
\hline 21 & 75 & $\mathrm{~F}$ & 17 & $\bullet$ & & & & & & & & & & $\bullet$ & & & & & $\bullet$ & & & & & $\bullet$ & & & & \\
\hline 22 & 64 & $M$ & 28 & $\bullet$ & & & & & $\bullet$ & & & & & $\bullet$ & & & & & $\bullet$ & & & & & $\bullet$ & & & & \\
\hline 23 & 73 & $M$ & 3 & $\bullet$ & $\bullet$ & $\bullet$ & & & & & & & & $\bullet$ & $\bullet$ & $\bullet$ & & & $\bullet$ & & & & & $\bullet$ & $\bullet$ & $\bullet$ & & \\
\hline 24 & 68 & $M$ & 27 & 0 & 0 & 0 & 0 & 0 & 0 & 0 & 0 & 0 & 0 & 0 & 0 & 0 & 0 & 0 & 0 & 0 & 0 & 0 & 0 & 0 & 0 & 0 & 0 & 0 \\
\hline 25 & 71 & $M$ & 0 & 0 & 0 & 0 & 0 & 0 & & & & & & & & & & & & & & & & 0 & 0 & 0 & 0 & 0 \\
\hline 26 & 66 & $M$ & 29 & 0 & 0 & 0 & 0 & 0 & 0 & 0 & 0 & 0 & 0 & 0 & 0 & 0 & 0 & 0 & 0 & 0 & 0 & 0 & 0 & 0 & 0 & 0 & 0 & 0 \\
\hline 27 & 63 & $M$ & 19 & 0 & 0 & 0 & 0 & 0 & 0 & 0 & 0 & 0 & 0 & 0 & 0 & 0 & 0 & 0 & $\bullet$ & $\bullet$ & & $\bullet$ & $\bullet$ & 0 & 0 & 0 & 0 & 0 \\
\hline 28 & 63 & $M$ & 22 & 0 & 0 & 0 & 0 & 0 & & & & & & 0 & 0 & 0 & 0 & 0 & 0 & 0 & 0 & 0 & 0 & 0 & 0 & 0 & 0 & 0 \\
\hline 29 & 81 & $F$ & 6 & 0 & 0 & 0 & 0 & 0 & & & & & & 0 & 0 & 0 & 0 & 0 & 0 & 0 & 0 & 0 & 0 & 0 & 0 & 0 & 0 & 0 \\
\hline 30 & 60 & $M$ & 25 & $\bullet$ & $\bullet$ & $\bullet$ & & & $\bullet$ & $\bullet$ & $\bullet$ & & & $\bullet$ & $\bullet$ & $\bullet$ & & & $\bullet$ & $\bullet$ & $\bullet$ & & & $\bullet$ & $\bullet$ & $\bullet$ & & \\
\hline
\end{tabular}

Data were express as follows: $\bigcirc$, all data was available at the 5 periods;, data at the 5 periods could not be assembled.

Figure 1- Profile of subjects receiving an esophageal cancer treatment and data obtained in each examination periods

\begin{tabular}{lcccccc}
\cline { 2 - 6 } Event: & \multicolumn{5}{c}{ operation } \\
POD (day): & $-7 \sim-4$ & $-4 \sim-3$ & 0 & $7 \sim 9$ & $10 \sim 15$ & $15 \sim 23$ \\
Examination: & E1 & E2 & \multicolumn{10}{c}{ E3 } & E4 & E5 \\
Nutrition: & reg. > reg. + suppl. $>$ NPO $>$ liquid $>$ semiliquid $>$ soft solids
\end{tabular}

Figure 2- Protocol of oral examination during the perioperative period of esophageal cancer surgery. Abbreviations are as follows: POD: postoperative day; reg.: regular meal; suppl.: additional enteral nutrient; NPO: Nil per os 
19 subjects at E2 and E3 were $0.52 \pm 0.30$ and $0.34 \pm 0.21 \mathrm{~mL} / \mathrm{min}$, respectively. This corresponds to a $35.3 \%$ reduction after operation. There were significant differences between E1 and E3 $(n=19$, $p<0.05), E 2$ and E3 $(n=19, p<0.01)$ and there were no significant differences between E2 and E4 $(n=18, p=0.06)$, E2 and E5 $(n=11, p=0.07)$. On the other hand, the mean of stimulated salivary flow rate \pm S.D. of 13 subjects of E2 and E3 were $2.74 \pm 0.82$ and $1.97 \pm 0.94 \mathrm{~mL} / \mathrm{min}$, respectively. There was a $28.1 \%$ reduction after operation. A significant difference regarding salivary flow of stimulated saliva was observed between E2 and E3 $(n=13, p<0.05)$ and E2 and E4 $(n=12, p<0.01)$. These results indicate that the salivary flow rate decreased significantly in the fasting period. The resting saliva secretion did not recover even when liquid food ingestion began.

\section{Alteration in clinical parameters}

PI and GI were used to evaluate plaque accumulation and gingival inflammation in subjects, respectively. There was a change in PI and GI for the pool of 10 subjects in the 5 examination periods
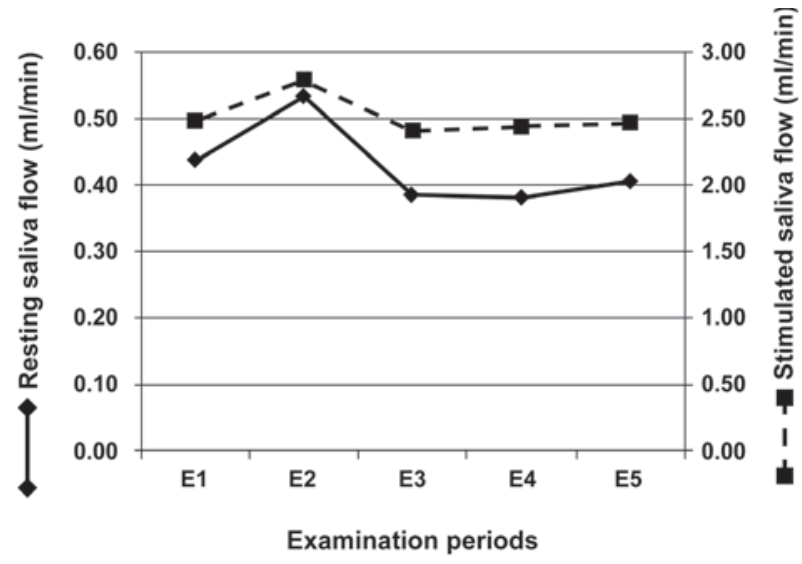

Figure 3- Fluctuations in saliva flow rate. The data represent the mean flow rate for resting saliva (solid line) and for stimulated saliva (broken line), respectively

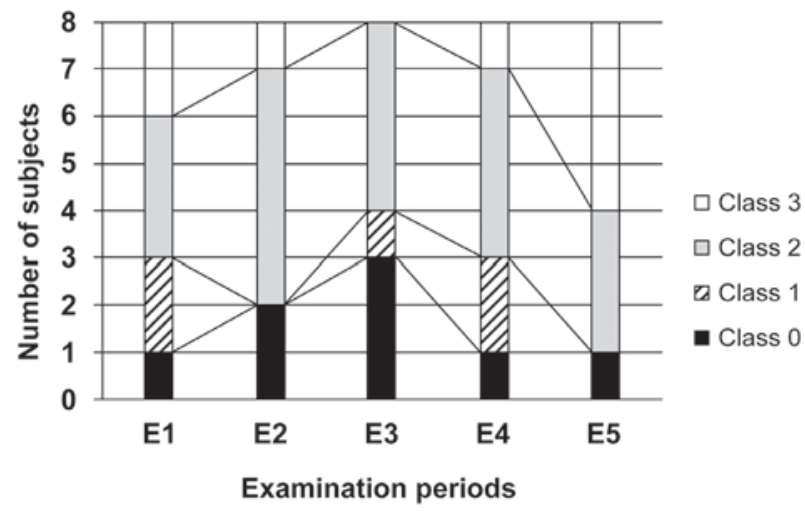

Figure 5- Distribution of the class of Dentocult SM Strip mutans in 8 subjects at the five examination points
(Figure 4). Regarding PI, there were significant differences between E1 and E3 $(n=18, p<0.05)$ and between E3 and E4 $(n=16, p<0.05)$, respectively. While there was no significant difference regarding GI, the same tendency for reduction of postoperative plaque accumulation was observed.

\section{Alteration in mutans streptococci levels in saliva}

We measured the concentration of mutans streptococci in saliva at each examination period using Dentocult SM kit by comparing the colony density on the test strip with the model chart (class 0 to class 3 ). The changes in mutans streptococci class from the pool of 8 subjects in the 5 examination periods is presented in Figure 5. There were significant differences between E2 and E3 $(n=14, p<0.05)$ and between E3 and E4 $(n=15$, $p<0.05)$, respectively. These results indicate that the colony-forming units of mutans streptococci decreased significantly through the fasting period. It increased significantly after the ingestion of semiliquid foods of rice porridge started.

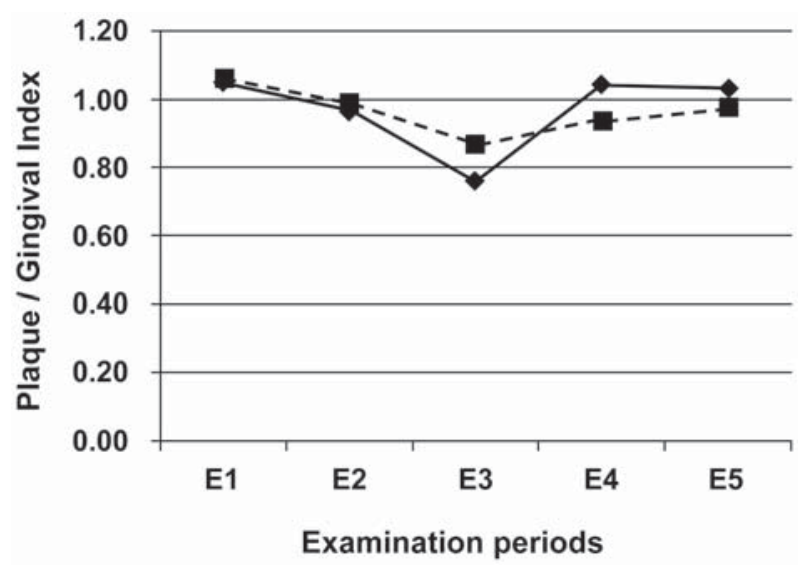

Figure 4- Alterations of plaque index ( $\mathrm{PI}$ : solid line) and gingival index (GI: broken line), respectively

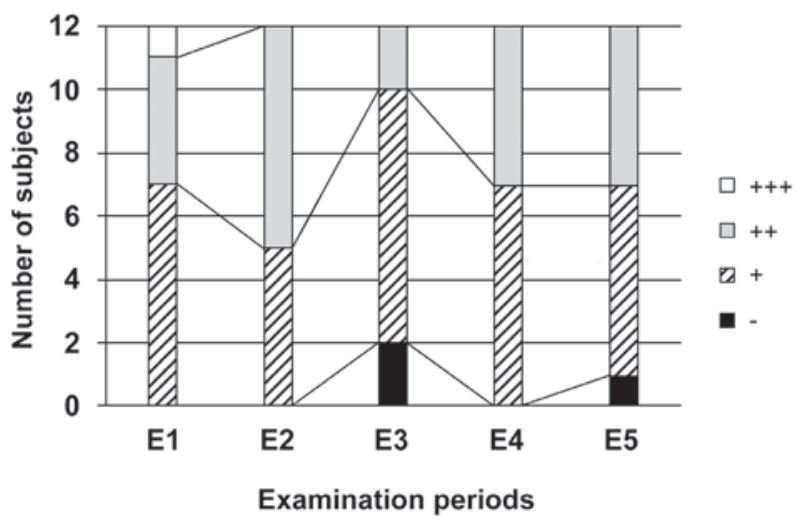

Figure 6- Distribution of the score of CAT21 test in 12 subjects at the five examination points 
Alteration in the acid production ability of dental plaque

Acid production ability of dental plaque was evaluated the using a CAT21 test at each examination period. Figure 6 shows the distribution of acid production levels for the pool of 12 subjects in the 5 examination periods. There was a significant difference between E2 and E3 $(n=19, p<0.05)$. The acid production level showed higher tendency after oral ingestion began, whereas there were no significant differences at E2 and E4. These results indicate that the acid production ability of dental plaque decreased significantly by deprived feeding during postoperative period and it recovered relatively quick after oral ingestion began.

\section{Alterations in repetitive saliva swallowing test}

We performed an RSST at bed-side. There were significant differences between E1 and E4 $(n=16$, $p<0.01)$ and between E2 and E4 $(n=18, p<0.05)$. The same tendency was observed between at E1 and E3, although without significant differences $(n=17, p=0.06)$. These results indicate that the state of swallowing function decreased significantly in the deprived feeding condition during the postoperative period.

\section{DISCUSSION}

In this study, we evaluated oral conditions during perioperative period at 5 points. Since subjects had just been hospitalized at $E 1$, eating and drinking habit were most likely specific for each patient and their oral conditions were modulated by their living habits. Therefore, we considered the E2 period as a control because all meals in hospital are standardized and the medical record could be used to follow the patients.

Although there is a great need for an oral health care clinical approach for esophagectomized patients during the perioperative period, very few information regarding the oral environment has been reported. Saliva is important for its buffering capacity and passive host immune defense against oral infections, including dental caries ${ }^{20}$. Salivary flow rate is related with bicarbonate concentration, which can act as a buffer ${ }^{9}$. We found in this study that salivary flow rate of both resting and stimulated saliva decreased significantly after NPO to $0.34 \mathrm{~mL} /$ $\mathrm{min}$ and $1.97 \mathrm{~mL} / \mathrm{min}$, respectively. They could not be steeply recovered after starting drinking and eating. Hyposalivation is usually defined by a flow rate of non-stimulated saliva less than $0.1 \mathrm{~mL} / \mathrm{min}$ or that of stimulated saliva less than $0.7 \mathrm{~mL} / \mathrm{ml}^{19}$. Although the values observed in this study were higher than the above criteria of hyposalivation, the low values of discrepancy between E2 and
E3 might influence the oral environment and be associated with discomfort related to the quality of life, such as eating, swallowing and speaking. Nakamura ${ }^{14}$ (1997) reported results from a mouse model indicating that short-term dietary mastication influenced the salivary activity and suggested that changes in autonomic nerves in the salivary glands occur rapidly depending on the sensitivity of the particular gland to masticatory stimulation. The reason for which the salivary flow rate of esophagectomized patients decreased significantly after NPO is unclear. However, at least for whole stimulated saliva, lack of main stimulators such as food chewing and tasting leads to depression of saliva secretion.

It has been demonstrated that pulmonary complications are the most common source of morbidity and mortality after esophagectomy ${ }^{2,13}$. Therefore, oral health care during the perioperative period is essential for the proper management of esophageal cancer. Interestingly, we found that PI decreases significantly after NPO, and that it can be recovered relatively quick compared with the salivary flow rate. The same tendency for caries activity test of mutans streptococci was observed. It was hypothesized that the number of oral bacteria having acid production ability decreased since the source of nutrient of plaque bacteria was drained by NPO for one week. For instance, the growth rate of mutans streptococci depends largely on carbohydrate concentration in the medium. Since sugar represents the main source of nutrients, the lack of it may have influenced strongly mutans streptococci. Furthermore, the antibiotic reagents prescribed for the patient for 3-4 days after the surgery likely affected the dental plaque including mutans streptococci at the time of E3 (POD7).

Swallowed foods have a high content of fermentable carbohydrates to support bacterial multiplication. It is thought that the reasons for which plaque accumulation increased significantly within several days after oral ingestion started (at E4) are related to: 1) the postoperative food form being soft, remaining stagnated in the mouth and becoming easily a substrate for dental plaque formation, 2) patients spent a long time at meal due to postoperative swallowing with functional depression, 3) increase of diet frequency due to ingestion of assistant foods, and 4) plaque accumulation is facilitated due to decrease of salivary flow rate. Although a significant difference was not observed, the tendency for reduction of postoperative GI and an increase of PI was observed at E3. This suggests a relationship between plaque accumulation and induction of gingival inflammation.

On the other hand, the swallowing function decreased significantly through NPO. However, the 
reason why subjects had a smaller number of times of swallowing at E4 cannot be clearly explained. It can be speculated that the prudence to swallowing operation would not become strong conversely at the patient itself because swallowing foods ingestion started, or the mental factor will act greatly. Postoperative factors associated with pulmonary complications include the aspiration or other abnormality of deglutition ${ }^{8}$. Upon identification of postesophagectomy swallowing abnormalities, various rehabilitation techniques may also be introduced to help prevent aspiration and subsequent respiratory complications, including the chin tuck and swallowing exercises ${ }^{3}$. Our results also indicated the recommendation of oral rehabilitation for prevention of respiratory complications.

We investigated alterations of the oral cavity in patients with esophageal cancer during the perioperative period. Prior to begin the study, we expected that both oral condition and function at NPO would get worse over time. However, plaque accumulation and mutans streptococci counts in saliva at NPO were better than before operation. On the other hand, oral hygiene was worsened relatively fast after the start of oral ingestion of liquid foods and it resulted in the recovery of oral functions such as saliva secretion and swallowing operation and getting better slowly. Ingestion of food is restricted after the esophagectomy, which means that the amounts of food have to decrease at meal and the frequency of meals has to increase. These eating habits may affect the oral hygiene of patients and increase the risks for dental caries and periodontal disease.

The importance of oral hygiene should be highlighted to esophageal cancer patients after esophagectomy. Yoneda, et al.22 (2007) reported that a professional oral care regimen in patients with esophageal cancer enabled the total population of oral streptococci microflora to remain stable and inhibited infections of the oral cavity by opportunistic pathogens, thereby inhibiting oral mucositis. In this study, mutans streptococci counts increased at the time of swallowing malfunction, which reinforces the fact that the oral conditions of the patients should be closely monitored in postoperative convalescence.

\section{CONCLUSIONS}

It may be concluded that it may be necessary a better management of the oral hygiene for maintaining a healthy oral environment in esophageal cancer patients. In addition, this could be applicable to many other types of patients that are hospitalized and do not eat and drink for a long time. Dental professionals should promote the salivary secretion and provide thorough instructions for plaque control for hospitalized patients.

\section{ACKNOWLEDGEMENTS}

We would like to thank Dr. Daniel Grenier (Laval University, Quebec, Canada) for editing this manuscript. This study was supported by Grant-inAid for Scientific Research 21592651 and 24390471 from Japan Society for the Promotion of Science.

\section{REFERENCES}

1- Akutsu Y, Matsubara H, Shuto K, Shiratori T, Uesato M, Miyazawa $Y$, et al. Pre-operative dental brushing can reduce the risk of postoperative pneumonia in esophageal cancer patients. Surgery. 2010;147:497-502.

2- Atkins BZ, D'Amico TA. Respiratory complications after esophagectomy. Thorac Surg Clin. 2006;16:35-48.

3- Atkins BZ, Fortes DL, Watkins KT. Analysis of respiratory complications after minimally invasive esophagectomy: preliminary observation of persistent aspiration risk. Dysphagia. 2007;22:49-54.

4- Baba Y, Teramoto S, Hasegawa H, Machida A, Akishita M, Toba $\mathrm{K}$. Characteristics and limitation of portable bedside swallowing test in elderly with dementia: comparison between the repetitive saliva swallowing test and the simple swallowing provocation test. Nihon Ronen Igakkai Zasshi. 2005;42:323-7.

5- Berti-Couto SA, Couto-Souza PH, Jacobs R, Nackaerts O, RibiraBullen IR, Westphalen FH. Clinical diagnosis of hyposalivation in hospitalized patients. J Appl Oral Sci. 2012;20:157-61.

6- Clarkson JE, Worthington HV, Eden TOB. Interventions for preventing oral candidiasis for patients with cancer receiving treatment (Review). Cochrane Database Syst Rev. 2009(4).

7- Daly JM, Lieberman MD, Goldfine J, Shou J, Weintraub F, Rosato $E F$, et al. Enteral nutrition with supplemental arginine, RNA, and omega-3 fatty acids in patients after operation: immunologic, metabolic, and clinical outcome. Surgery. 1992;112:56-67.

8- D'Amico TA. Improving outcomes after esophagectomy: the importance of preventing postoperative pneumonia. Zhonghua Wei Chang Wai Ke Za Zhi. 2011;14:660-6.

9- Fenoll-Palomares C, Muñoz Montagud JV, Sanchiz V, Herreros $B$, Hernández V, Mínguez $M$, et al. Unstimulated salivary flow rate, $\mathrm{pH}$ and buffer capacity of saliva in healthy volunteers. Rev Esp Enferm Dig. 2004;96:773-83.

10- Fujita T, Daiko H, Nishimura M. Early enteral nutrition reduces the rate of life-threatening complications after thoracic esophagectomy in patients with esophageal cancer. Eur Surg Res. 2012;48:79-84.

11- Loe H. Silness J. Periodontal disease in pregnancy I. Prevalence and severity. Acta Odontol Scand. 1963;21:533-51.

12- Matsumoto S, Koga H, Kusaka J, Hagiwara S., Shihara K, Kai $S$, et al. Effects of the antioxidant-enriched concentrated liquid diet ANOM on oxidative stress and multiple organ injury in patients with septic shock: a pilot study. J Anesthe Clinic Res. 2011,2:155. 13- McKevith JM, Pennefather SH. Respiratory complications after oesophageal surgery. Curr Opin Anaesthesiol. 2010;23:34-40.

14- Nakamura K. Effects of short-term bulk or liquid diet feeding on the neurotransmitters in the salivary glands and various sialogogue-induced salivation in mice. Jpn J Oral Biol. 1997;39:655-64.

15- Pavia R, Barresi P, Piermanni V, Mondello B, Urgesi R. Role of artificial nutrition in patients undergoing surgery for esophageal cancer. Rays. 2006;31:25-9.

16- Sato M, Yoshihara A, Miyazaki H. Preliminary study on the effect of oral care on recovery from surgery in elderly patients. J Oral Rehabil. 2006;33:820-6. 
17- Seike J, Tangoku A, Yuasa Y, Okitsu H, Kawakami Y, Sumitomo $M$. The effect of nutritional support on the immune function in the acute postoperative period after esophageal cancer surgery: total parenteral nutrition versus enteral nutrition. J Med Invest. 2011; 58:75-80.

18- Silness J, Loe H. Periodontal disease in pregnancy. II. Correlation between oral hygiene and periodontal condition. Acta Odontol Scand. 1964;22:121-35.

19- Von Bultzingslowen I, Sollecito TP, Fox PC, Daniels T, Jonsson $R$, Lockhart $P B$, et al. Salivary dysfunction associated with systemic diseases: systematic review and clinical management recommendations. Oral Surg Oral Med Oral Pathol Oral Radiol Endod. 2007;103 (Suppl 1):S57 e1-e15.
20- Wolinsky LE. Caries and cariology. In: Nisengard RJ, Newman MG. Oral microbiology and immunology. $2^{\text {nd }}$ ed. Philadelphia: W. B. Saunders; 1994. p. 341-59.

21- Worthington HV, Clarkson JE, Eden TOB. Interventions for preventing oral mucositis for patients with cancer receiving treatment (Review). Cochrane Database Syst Rev. 2011(4): CD000978.

22- Yoneda S, Imai S, Hanada N, Yamazaki T, Senpuku H, Ota Y, et al. Effects of oral care on development of oral mucositis and microorganisms in patients with esophageal cancer. Jpn J Infect Dis. $2007 ; 60: 23-8$. 